\title{
The high-affinity transporter BnPHT1;4 is involved in phosphorus acquisition and mobilization for facilitating seed germination and early seedling growth of Brassica napus
}

\author{
Ke-Lin Huang ${ }^{\dagger}$, Huan Wang ${ }^{\dagger}$, Ying-Li Wei, Han-Xin Jia, Lei Zha, Yong Zheng, Feng Ren ${ }^{*}$ and Xue-Bao Li ${ }^{*}$ (D)
}

\begin{abstract}
Background: Seed germination and seedling establishment are two of the most critical phases in plant development. However, the molecular mechanisms underlying the effect of phosphorus on seed germination and post-germinated growth of oilseed rape are unclear so far. Here, we report the role of BnPHT1;4 in seed germination and early seedling development of Brassica napus.

Results: Our results show that BnPHT1;4 is preferentially expressed in cotyledons of early developing seedlings. Overexpression of BnPHT1;4 in oilseed rape promoted seed germination and seedling growth. Expression levels of the genes related to $A B A$ and GA biosynthesis and signaling were significantly altered in BnPHT1;4 transgenic seedlings. Consequently, active GA level was up-regulated, whereas ABA content was down-regulated in BnPHT1;4 transgenic seedlings. Furthermore, exogenous GA could promote seed germination of wild type, while exogenous ABA could partially recover the advanced-germination phenotype of BnPHT1;4 transgenic seeds. Total phosphorus content in cotyledons of the transgenic seedlings was decreased more rapidly than that in wild type when Pi was supplied or deficient, and Pi contents in shoots and roots of the BnPHT1;4 transgenic plants were higher than those in wild type under high and low Pi conditions.

Conclusions: Our data suggest that the high-affinity transporter BnPHT1;4 is involved in phosphorus acquisition and mobilization for facilitating seed germination and seedling growth of Brassica napus by modulating ABA and GA biosynthesis.
\end{abstract}

Keywords: Brassica napus, Phosphorus (P), Phosphate (pi), Abscisic acid (ABA), gibberellin (GA), Seed germination, Seedling growth and development

\section{Background}

Seed germination is one of the most critical phases for the establishment of a plant [1]. A seed achieves germination when the appropriate nutrients conditions are present. Nutrient provision in seed germination and early seedling development only depends on storage products deposited in cotyledons at the end of seed (embryo) development

\footnotetext{
*Correspondence: xbli@mail.ccnu.edu.cn; renfeng@mail.ccnu.edu.cn ${ }^{+}$Ke-Lin Huang and Huan Wang are contributed equally to this work. Hubei Key Laboratory of Genetic Regulation and Integrative Biology, School of Life Sciences, Central China Normal University, Wuhan 430079, China
}

before the root system have been established [2]. Previous studies revealed the influence of nutrients on seed germination. For instance, $\mathrm{C}$ and $\mathrm{N}$ control seed germination through interacting with phytohormones [3]. S-metabolism and ABA biosynthesis co-regulate seed germination of Arabidopsis thaliana [4]. $\mathrm{K}$ participates in maintaining turgor stability and osmoregulation of cells [5]. $\mathrm{Zn}, \mathrm{Fe}, \mathrm{Cu}, \mathrm{Ni}$, $\mathrm{Mo}, \mathrm{Mn}, \mathrm{B}$ and $\mathrm{Cl}$ take the structural roles in stabilizing proteins or act as catalytically active cofactors in enzymes [6]. However, little is known about the link of phosphorus nutrients and seed germination so far.

(C) The Author(s). 2019 Open Access This article is distributed under the terms of the Creative Commons Attribution 4.0 International License (http://creativecommons.org/licenses/by/4.0/), which permits unrestricted use, distribution, and 
Phosphorus (P), the composition of membranelipids, energy carrier (ATP) and heredity (DNA and RNA), is an essential macro-element for plant growth and development [7-9]. Although a large amount of phosphorus exists in soil, the phosphate $(\mathrm{Pi})$ readily available for plants is insufficient in soil [7]. Pi concentration within plant cells is usually above $10 \mathrm{mM}(>3 \mathrm{~g} / \mathrm{kg})$, but the concentration of Pi in most of the soil is typically lower than $10 \mu \mathrm{M}$ [10]. Plants have evolved both high and low-affinity Pi transporters that mediate both $\mathrm{P}$ uptake and translocation in plants [11]. These Pi transporters belong to four subfamilies, PHT1 (phosphate transporter) to PHT4, and are plasma membrane proteins that are proton-coupled $\mathrm{H}_{2} \mathrm{PO}_{4}{ }^{-}$symporters [12]. It was suggested that the high-affinity PHT1 family transporters are responsible for acquiring $\mathrm{P}$ from rhizospheres [13]. Unlike PHT1, proteins of the PHT2, PHT3 and PHT4 families are organelle Pi transporters and symport Pi into or out plastid (PHT2/4), mitochondrial (PHT3), or Golgi (PHT4) membranes [13]. Besides, some Pi transporters (such as AtPHT1;5) plays a critical role in mobilizing $\mathrm{P}$ between source and sink organs, and mobilizing the stored $\mathrm{P}$ out of older leaves crucial to maintain $\mathrm{P}$ homeostasis [14]. Expression analysis of the PHT1 family members indicated that AtPHT1;1, AtPHT1;3, AtPHT1;4, AtPHT1;5, and AtPHT1;6 are expressed in the hydathodes of cotyledons, suggesting a $\mathrm{P}$ transport system existed in cotyledons [15]. Histochemical assay revealed that strong GUS activity under the control of AtPHT1;4 and AtPHT1;5 promoters was detected throughout the cotyledons, suggesting AtPHT1;4 and AtPHT1;5 may be responsible for P mobilization in cotyledons [15]. Moreover, it has been reported that alteration of OsPHT1;4 expression affected embryo development and had an effect on seed germination ability and subsequent seedling growth of rice [16].

Previous studies have extensively shown that phytohormones gibberellins (GA) and abscisic acid (ABA) are two of the primary endogenous factors regulating seed germination antagonistically. GA promotes the transition from seed dormancy to germination, whereas ABA inhibits this process [17-20]. In Arabidopsis, for instance, the impaired germination phenotype of seeds is observed in mutants ( $g a 1$ and $g a 2$ ) that are severely compromised in GA biosynthesis, while the loss-of-function of GA2OX2 ( $g a 2 o \times 2 \mathrm{mu}$ tation) causes an increase in GA4 levels and promotes the germination of the mutant seeds [21, 22]. In contrast to GA mutants, seeds of the ABA-deficiency mutants (nced3, $n c e d 5, n c e d 6$ and $a b a 2$ ) exhibit a faster-germination phenotype in Arabidopsis [23-25]. Furthermore, the ABA signaling mutants (abi3, abi4 and abi5) of Arabidopsis showed the decreased seed dormancy and faster-germinating phenotypes $[21,26]$. However, overexpression of the enhancer $A B I 4$ that is involved in ABA signal pathway inhibited seed germination of Arabidopsis [21]. Similarly, tobacco $A B A 2$ overexpression transgenic seeds exhibited the delayed- germination phenotype [27]. It has been indicated that the balance of $\mathrm{ABA}$ and GA determines the fate of a seed, dormancy or germination [19]. Additionally, N, P and S nutrients may be connected with hormone signaling during seed germination $[3,4]$.

In this study, we aim to reveal the possible phosphorus transport mechanism in cotyledons of Brassica napus. Our data indicated that expression level of BnPHT1;4 is significantly higher than that of BnPHT1;5 in cotyledons, so we focused on investigating the role of BnPHT1;4 in early seedling development of Brassica napus. Although previous study reported the BnPHT1;4 was involved in phosphorus uptake and roots architecture in the transgenic Arabidopsis plants [28], large amount of work is needed to dissect the biological roles of BnPHT1;4 in Brassica napus. Our results revealed that overexpression of BnPHT1;4 promotes seed germination and seedling growth of Brassica napus possibly by modulating ABA and GA biosynthesis. BnPHT1;4 is involved in phosphorus mobilization in cotyledons and phosphorus uptake from soil in roots during early seedling growth, indicating that BnPHT1;4 plays a critical role in phosphorus acquisition and mobilization for facilitating seed germination and early seedling growth of Brassica napus.

\section{Results}

BnPHT1;4 is predominantly expressed in the cotyledons of Brassica napus

To reveal the possible phosphorus transport mechanism in cotyledons of $B$. napus, we first analyzed the expression levels of BnPHT1;4 and BnPHT1;5 in early developing seedlings of $B$. napus, and found that BnPHT1;4 expression is significantly higher than that of BnPHT1;5 during seed germination (Additional file 1 Figure S1). Then, we further analyzed expression of BnPHT1;4 in tissues (such as cotyledons, hypocotyls and roots) at early seedling developmental stage. As shown in Fig. 1a, BnPHT1;4 was predominantly expressed in cotyledons during early seedling development. To further elucidate the tissue-specific/preferential expression pattern of BnPHT1;4, we generated transgenic $B$. napus plants carrying a transcriptional fusion of the BnPHT1;4 promoter and the reporter GUS gene. Histochemical assay revealed that GUS activity under the control of BnPHT1;4 promoter was mainly detected in cotyledons, especially in very early seedling developmental stage after seed germination. As seedlings further developed, GUS activity was dramatically decreased in cotyledons, while no or very weak GUS staining was detected in roots and hypocotyls (Fig. 1b-e). Quantitative RT-PCR analysis also indicated that expression of the GUS reporter gene under the control of the BnPHT1;4 promoter was mainly in cotyledons (Fig. 1f), suggesting that BnPHT1;4 promoter is very active in cotyledons of the early developmental seedlings. Additionally, BnPHT1;4 expression reaches to its peak 

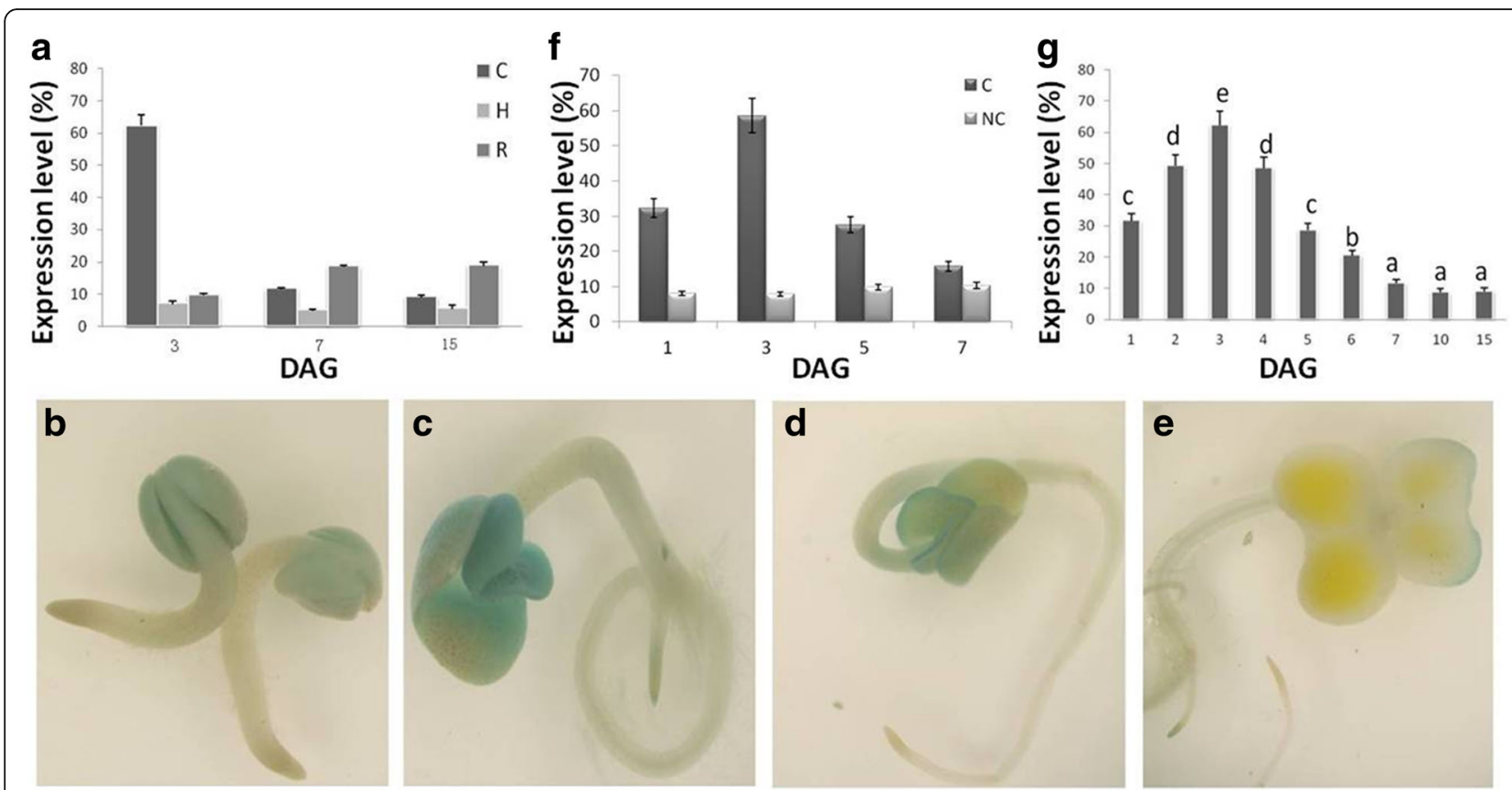

Fig. 1 Expression profiling of BnPHT1;4 in early developing seedlings of Brassica napus. (a) Quantitative RT-PCR analysis of BnPHT1;4 expression in oilseed rape seedlings at 3-15 DAG. Total RNA was isolated from roots (R), hypocotyls (H), cotyledons (C) of seedlings cultured in normal conditions (1 $\mathrm{mM} \mathrm{Pi}$ ) at 3, 7 and 15 DAG for RT-PCR analysis, using BnACT2 gene as standard control. The error bars indicate the standard errors $(n=3)$. (b-e). Histochemical assay of GUS activity in early developing seedlings. GUS staining observation of transgenic $B$. napus plants harboring the $B n P H T 1 ; 4$ promoter: GUS fusion gene in 1 DAG (b), 3 DAG (c), 5 DAG (d) and 7 DAG (e) seedlings cultured on MS medium with 1 mM Pi, respectively. (f) Quantitative RT-PCR analysis of the GUS gene in cotyledons (C) and in roots plus leaves and hypocotyls (NC) of BnPHT1;4 promoter: GUS transgenic plants. Total RNAs were isolated from C and NC of 1 DAG, 3 DAG, 5 DAG and 7 DAG seedlings cultured on MS medium with 1 mM Pi, respectively, for RT-PCR analysis, using BnACT2 gene as standard control. (g) Quantitative RT-PCR analysis of BnPHT1;4 expression in cotyledons of oilseed rape seedlings at 1-15 DAG. Total RNAs was isolated from cotyledons of seedlings cultured in normal conditions (1 mM Pi) at 1-15 DAG for RT-PCR analysis, using BnACT2 gene as standard control. The error bars indicate the standard errors $(n=9)$. Significance of difference was analyzed by Duncan's test $(P<0.05)$. Different lowercase letters indicate statistically significant differences. DAG, day after germination

value in cotyledons at three days after seed germination (DAG), and thereafter, gradually declined in cotyledons (Fig. 1g). Collectively, these results suggested that BnPHT1;4 may play an important role in cotyledons during seed germination and early seedling development.

\section{BnPHT1;4 is induced in cotyledons and roots under phophorus deficiency}

BnPHT1;4 is induced in B. napus under phosphorus (P) deficiency [28]. To further characterize BnPht1;4 response to $\mathrm{P}$ concentrations during seed germination and seedling growth, we employed quantitative RT-PCR analysis to determine the expression of BnPHT1;4 in roots and cotyledons at early developmental stages of seedlings under different $\mathrm{Pi}$ conditions. The expression of BnPHT1;4 in both roots and cotyledons was not responsive to P deprivation before 3 DAG, but BnPHT1;4 expression was dramatically induced under P-starvation condition after 3 DAG in both cotyledons and roots (Fig. 2a-b). Moreover, pre-germinated seedlings were grown for $15 \mathrm{~d}$ on P-sufficient medium, and then transferred to medium with different $\mathrm{Pi}$ concentrations for 3d. BnPHT1;4 expression in roots and cotyledons of these seedlings were analyzed by RT-PCR. As shown in Fig. 2c-d, transcriptional tendency of BnPHT1;4 were similar to 5 DAG that BnPHT1;4 is induced in cotyledons and roots under $\mathrm{P}$ deficiency. Moreover, pre-germinated seedlings grew on P-sufficient medium for 21 days, and then transferred to medium with $1 \mathrm{mM}$ Pi or $20 \mu \mathrm{M}$ Pi for 3 days. BnPHT1;4 expression in roots and shoots of these seedlings were analyzed by quantitative RT-PCR. As shown in Additional file 1 Figure S2, expression level of BnPHT1;4 was remarkably enhanced in both shoots and roots under P deficiency, relative to that under P-sufficient condition. These results indicate that $B n P H T 1 ; 4$ is induced in cotyledons and roots of $B$. napus under P deprivation.

\section{Overexpression of $B n P H T 1 ; 4$ in $B$. napus affects seed germination and seedling growth}

To characterize the function of BnPHT1;4 in P uptake and translocation in $B$. napus, we generated $B n P H T 1 ; 4$ overexpression transgenic $B$. napus plants $(\mathrm{OEBnPHT1;4)}$ by Agrobacterium tumefaciens-mediated transformation. We 

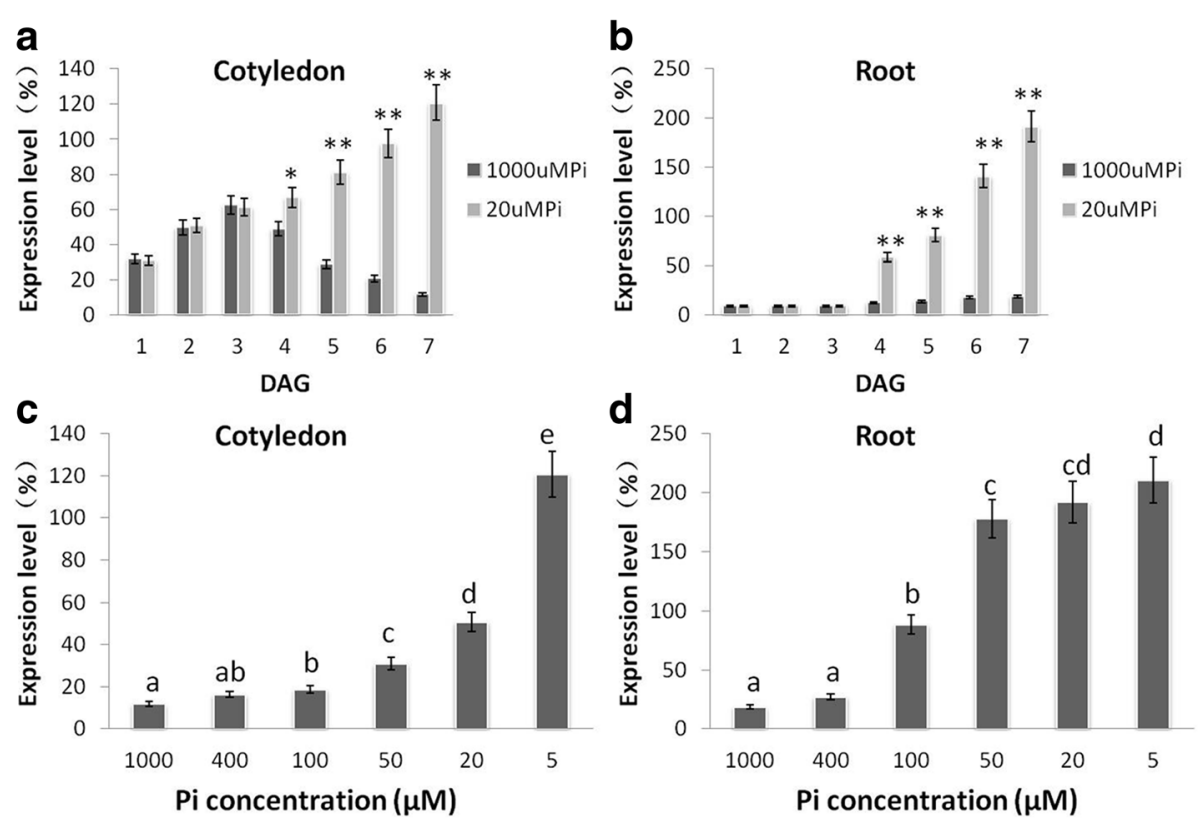

Fig. 2 Expression profiling of BnPHT1;4 in cotyledons and roots of Brassica napus under Pi starvation. (a and $\mathbf{b}$ ) Quantitative RT-PCR analysis of BnPHT1;4 expression in cotyledons (a) and roots (b) of oilseed rape seedlings at 1-7 DAG. Total RNA was isolated from cotyledons (a) and roots (b) of seedlings under 1000 or $20 \mu \mathrm{M}$ Pi at 1-7 DAG for RT-PCR analysis, using BnACT2 gene as standard control. (c and d) Quantitative RT-PCR analysis of BnPHT1;4 expression in cotyledons (c) and roots (d) of oilseed rape seedlings under different Pi concentrations. The 7 DAG seedlings were transferred to 5, 20,50,100, 400 or $1000 \mu \mathrm{M}$ Pi for 3 days, and then total RNA was isolated from cotyledons (c) and roots (d) for RT-PCR analysis, using BnACT2 gene as standard control. $\mathrm{KCl}$ was used to replace $\mathrm{KH}_{2} \mathrm{PO}_{4}$ in the medium for the equivalent amount of potassium. The error bars indicate the standard errors. Significance of difference was analyzed by Duncan's test $\left({ }^{*} p<0.05,{ }^{* *} p<0.01, n=3\right)$. Different lowercase letters indicate statistically significant differences. DAG, day after germination

selected four lines OEBnPHT1;4-5 (S5), OEBnPHT1;4-9 (S9), OEBnPHT1;4-10 (S10) and OEBnPHT1;4-11 (S11) for further characterization. The expression levels of BnPHT1;4 were increased by 3- to 6-folds in OEBnPHT1;4 lines compared with those in the wild type (WT) and transgenic null line (CK) (Fig. 3a).

We then examined seed germination of these four independent OEBnPHT1;4 lines under P-sufficient (1 mM) conditions, using WT and CK as controls. As shown in Fig. 3b, OEBnPHT1;4 seeds germinated at a faster rate than control seeds. We determined the resulting germination frequencies by counting the number of seeds with protruding radicles. The results indicated that seed germination of OEBnPHT1;4 lines is faster than the controls (Fig. 3c). Similarly, the length of hypocotyls and primary roots of the transgenic seedlings was significantly increased, relative to that of the controls (Fig. 3d-i). However, overexpression of BnPHT1;4 don't affect the total P content and weight of rape seeds (Additional file 1 Figure S3 and Figure S4).

\section{Overexpression of BnPHT1;4 enhances GA biosynthesis, but suppresses ABA biosynthesis}

Previous studies demonstrated that ABA and GA play key roles in seed germination $[17,18]$. Therefore, we further investigated whether the effect of BnPHT1;4 on seed germination is related to GA and ABA pathways. We compared the dynamic changes in expression of genes involved in ABA and GA biosynthesis and signaling in the transgenic lines and wild type controls of $B$. napus during the imbibition process of seeds. Quantitative RT-PCR analysis showed that the expression levels of the ABA biosynthesis-related genes BnNCED9 and BnNCED5 were significantly decreased in the BnPHT1;4 transgenic lines. Also, expression of $B n A B I 4$ was declined in the transgenic lines, relative to wild type (Fig. 4). Subsequently, we examined the expression patterns of the key genes involved in GA biosynthesis and signaling pathway. The results showed that expression of $B n G A 1, B n G A 2, B n G A 3$ and $B n K A O$ was enhanced in the BnPHT1;4 transgenic lines at 3 to $12 \mathrm{~h}$ after imbibitions of seeds, compared with those in wild type (Fig. 4), suggesting BnPHT1;4 probably positively regulates GA biosynthesis during seed germination of $B$. napus. Consequently, we determined the endogenous $\mathrm{ABA}$ and active GA levels during imbibition of seeds. The results indicated that overexpression of BnPHT1;4 significantly decreased active ABA levels in the imbibed transgenic seeds (Fig. 5a). In contrast, the GA1, GA3, and GA4 concentrations in the imbibed transgenic seeds were remarkably increased during imbibition, 

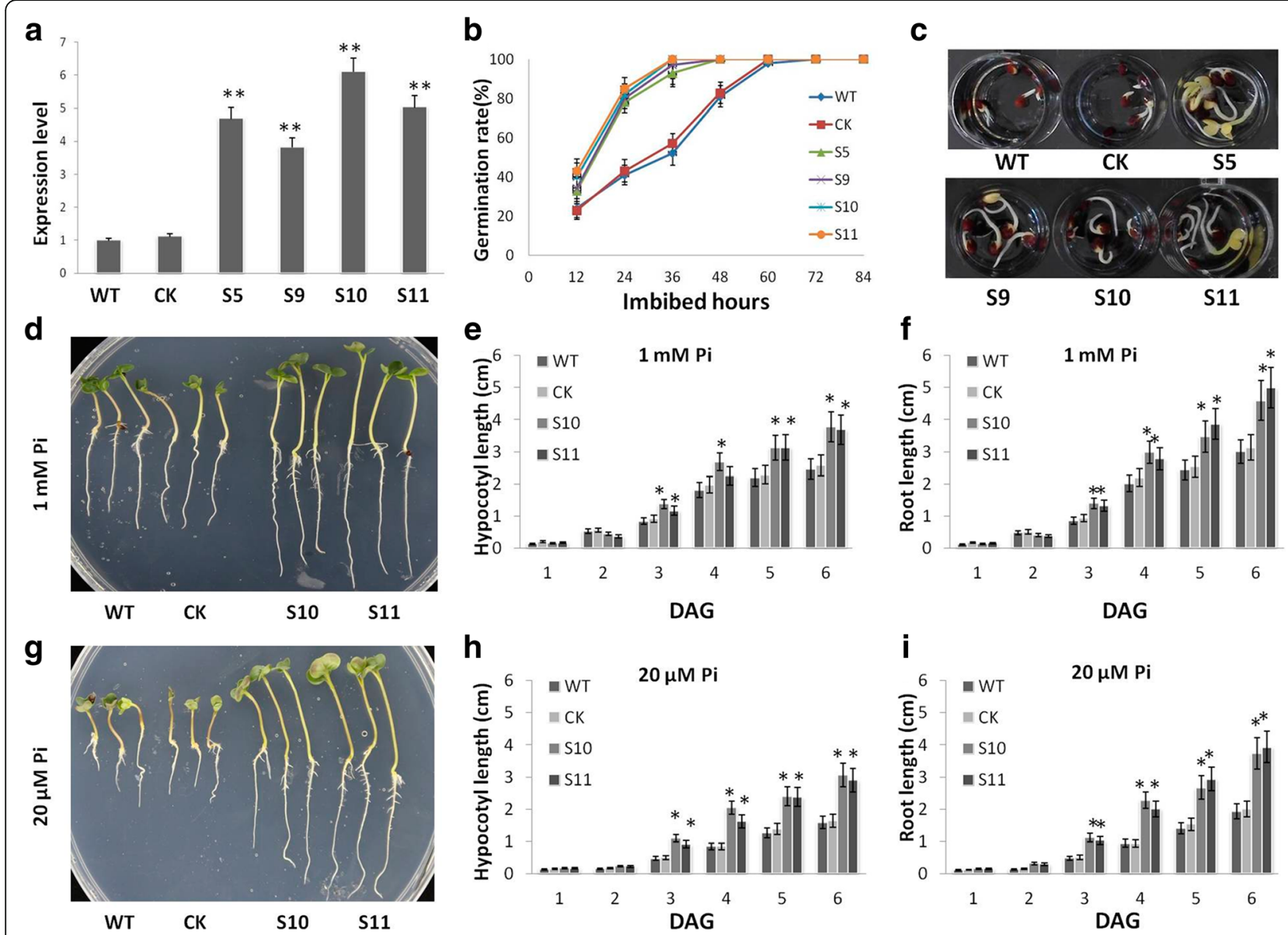

Fig. 3 BnPHT1;4 promotes seed germination and seedling growth of Brassica napus. (a) Detection of the transcript abundance of BnPHT1;4 in the BnPHT1;4 overexpression transgenic seedlings and controls (WT and CK) by quantitative RT-PCR analysis. Total RNA was isolated from roots and shoots of the 7 DAG seedlings. Expression levels of the BnPHT1;4 in wild type are set to 1, and in the transgenic lines are shown in folds compared with that in wild type. The error bars indicate the standard errors. Significance of difference was analyzed by Duncan's test $\left({ }^{*} p<0.05,{ }^{* *} p<0.01, n=3\right.$ ). (b and $\left.\mathbf{c}\right)$ Overexpression of BnPHT1;4 promotes seed germination. Seed germination of the BnPHT1;4 overexpression transgenic lines and controls on 1/2 MS medium with $1000 \mu \mathrm{M}$ Pi. Seeds were stored for 2 months after harvest and subjected to analysis. After seeds were sowed, quantitative analysis of germination rates at different time (hours of seed imbibition) are shown in b, and one representative image per genotype (1.5 days after sowing) is shown in c. (d-i) Overexpression of BnPHT1;4 promotes early seedling growth. Seeds of the controls were sowed about $24 \mathrm{~h}$ earlier than those of BnPHT1;4 transgenic lines, and then the seedlings with same length of radicle grew on 1/2 MS medium with 20 or $1000 \mu \mathrm{M} \mathrm{Pi}$. $\mathrm{KCl}$ was used to replace $\mathrm{KH}_{2} \mathrm{PO}_{4}$ in the medium for the equivalent amount of potassium. One representative image per genotype (1.5 days after sowing) is shown in $\mathrm{d}(1000 \mu \mathrm{M}$ $\mathrm{Pi})$ and $\mathrm{g}(20 \mu \mathrm{M} \mathrm{Pi})$. Quantitative analysis of hypocotyl length and root length in $1000 \mu \mathrm{M}$ Pi are shown in e and f, while in $20 \mu \mathrm{M} P \mathrm{Pi}$ are shown in $\mathrm{h}$ and i. Five experimental replications were performed and each replication contains at least 150 seedlings. The data are presented as means \pm SD $(n=5)$. WT, wild type; CK, transgenic null line; S5, S9, S10 and S11, four independent BnPHT1;4 overexpression transgenic lines. DAG, day after germination

compared with those in the controls (Fig. 5b-d), and ratios of GA1/ABA, GA3/ABA and GA4/ABA were up-regulated (Fig. 5e). Altogether, the results suggest that BnPHT1;4 may negatively modulate ABA biosynthesis but positively regulates GA biosynthesis in B. napus.

To further confirm the role of BnPHT1;4 in GA and $A B A$ biosynthesis during seed germination, we tested the responsiveness of rape seeds to $A B A, G A$ and paclobutrazol (PAC, a GA biosynthesis inhibitor) during seed imbibition. We supposed that the advanced-germination phenotype of the BnPHT1;4 overexpression transgenic seeds is caused by the enhanced GA biosynthesis. The experimental results indicated that PAC could partially delay the advancedgermination phenotype of the BnPHT1;4 transgenic seeds. The effects on post-germination growth parameters, such as radical length, were also similar to the germination results. Furthermore, exogenous GA could narrow the gap of seed germination velocity between the transgenic lines and controls (Fig. 6a-b and Additional file 2 Dataset S4). On the other hand, exogenous $\mathrm{ABA}$ inhibited seed germination of both 

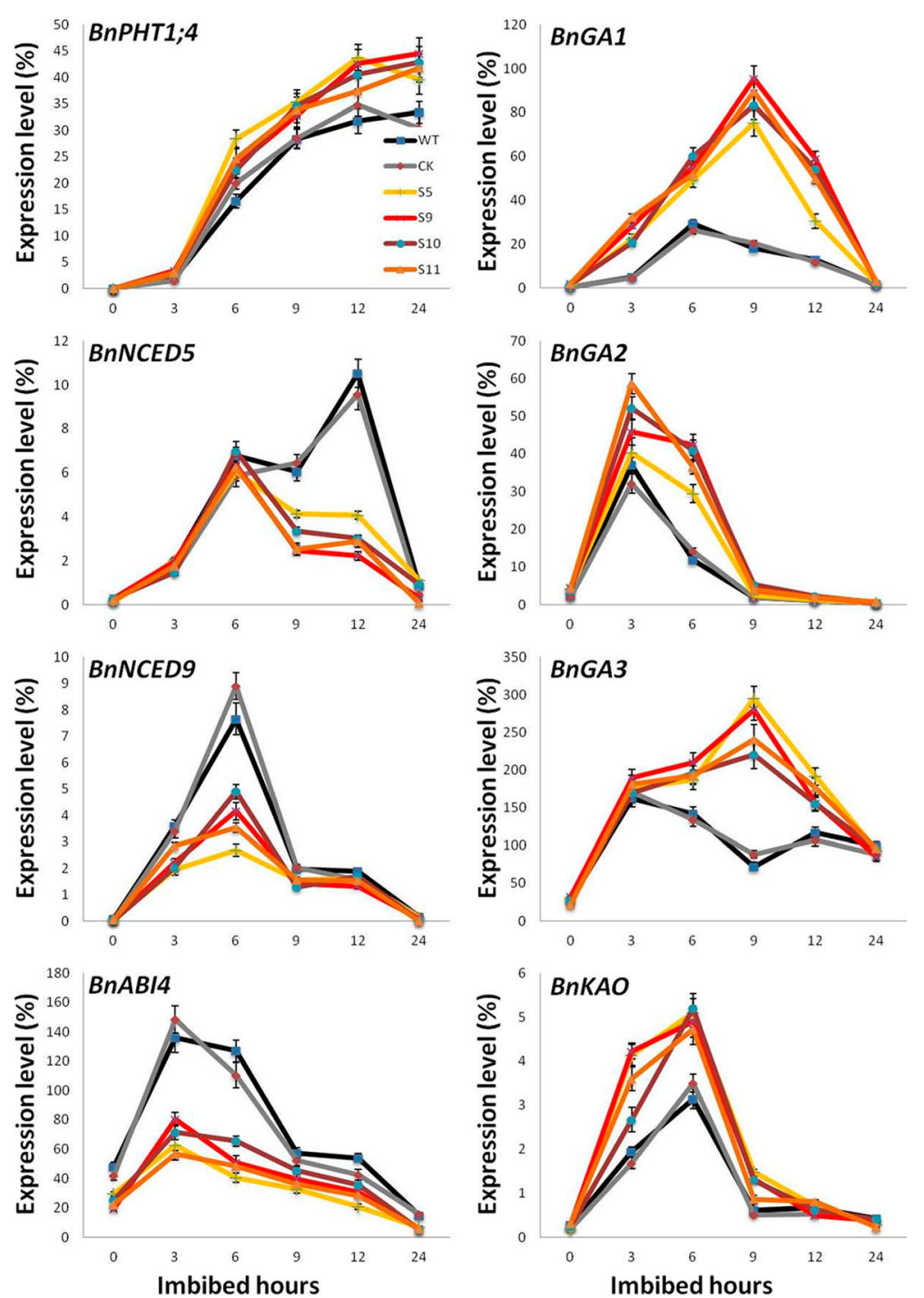

Fig. 4 Quantitative RT-PCR analysis of gene expressions in dry and imbibed seeds of the BnPHT1;4 overexpression transgenic Brassica napus. Gene expression of BnPHT1;4, GA-related genes (BnGA1, BnGA2, BnGA3 and BnKAO1), ABA biosynthesis-related genes (BnNCED5 and BnNCED9) and ABA signaling positive regulator gene $(B \cap A B / 4)$ was determined in germinating seeds during the course of the imbibition process. Two-month stored seeds were used for seed germination, and total RNA was isolated from the dry and imbibed (germinating) seeds for RT-PCR analysis, using BnACT2 gene as standard control. The data are presented as means \pm SD $(n=3)$, and significance of difference was analyzed by Duncan's test. WT, wild type; CK, transgenic null line; S5, S9, S10 and S11, four independent BnPHT1;4 overexpression transgenic lines

transgenic lines and wild type controls, and reduced the index of germination velocity of the transgenic seeds and controls. Moreover, exogenous ABA could partially weaken the effect caused by overexpression of BnPHT1;4. This trend was more pronounced at higher $\mathrm{ABA}$ concentrations, especially when the $\mathrm{ABA}$ concentrations reach to $100 \mu \mathrm{M}$ (Fig. 6c-d and Additional file 2 Dataset S3). Taken together, these results revealed that overexpression of BnPHT1;4 promotes seed germination process of $B$. napus by suppressing ABA biosynthesis while promoting GA biosynthesis.

\section{BnPHT1;4 is involved in phosphorus mobilization in} seedlings and phosphorus uptake from soil

As BnPHT1;4 is abundantly expressed in cotyledons, and overexpression of BnPHT1;4 in B. napus affects seed germination, we deduce that BnPHT1;4 may be involved in $\mathrm{P}$ mobilization in cotyledons that supply $\mathrm{P}$ for the formation of new organs. So, we analyzed total phosphorus contents in cotyledons and non-cotyledons (including roots, hypocotyls and new leaves) at the stages of seed germination and early seedling growth. As shown in Fig. 7a and Additional file 2 Dataset S1, total phosphorus 

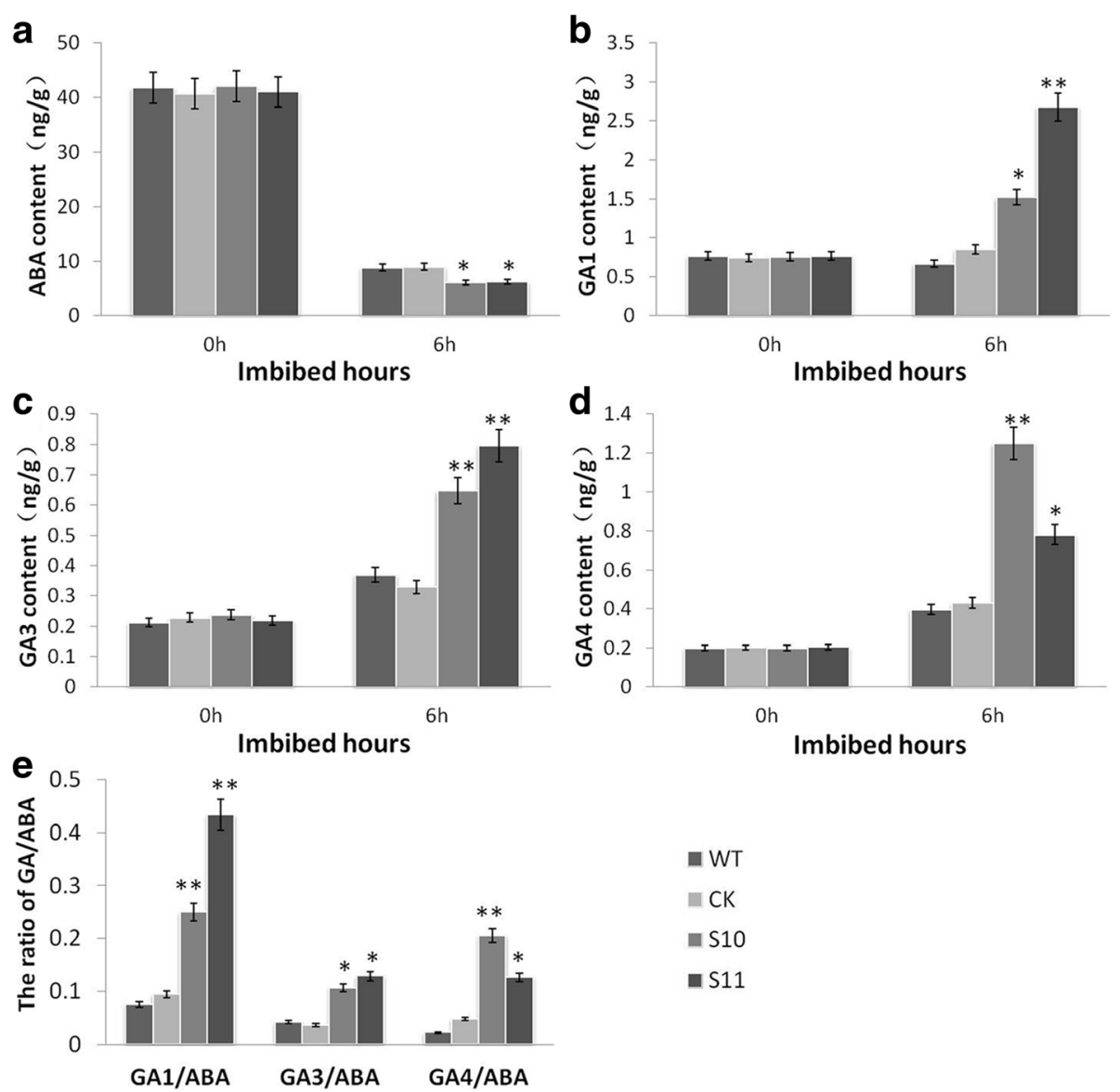

Fig. 5 Assay of endogenous GA and ABA contents in BnPHT1;4 overexpression transgenic seeds of Brassica napus. (a-d) Contents of ABA, GA1, GA3 and GA4 in dry and imbibed seeds of the transgenic lines (S10 and S11) and controls (WT and CK) (see Methods). (e) Ratios of GA1/ABA, GA3/ABA, and GA4/ $A B A$ in dry and imbibed seeds of the transgenic lines and controls. The error bars indicate the standard errors. Significance of difference was analyzed by Duncan's test ( ${ }^{*} p<0.05$, ${ }^{* *} p<0.01, n=3$ ). WT, wild type; CK, transgenic null line; S10 and S11, independent BnPHT1;4 overexpression transgenic lines

was constantly decreased in cotyledons of both OEBnPHT1;4 lines and controls. Moreover, total phosphorus content in cotyledons of transgenic plants was declined more rapidly than that in the wild type and CK controls. To further demonstrate the role of BnPHT1;4 in phosphorus mobilization in cotyledons, we analyzed total phosphorus dynamics during seed germination and early seedling growth without exogenous Pi supply (Fig. 7b and Additional file 2 Dataset S2). In the absence of exogenous phosphorus, total phosphorus content in cotyledons was decreased more rapidly in both transgenic plants and controls, suggesting that there is a mechanism of phosphorus transport in cotyledons response to phosphorus change. Furthermore, total phosphorus content in the transgenic plants was decreased faster than that in the controls when no exogenous $\mathrm{Pi}$ was supplied in solution, indicating BnPHT1;4 may play an important role in phosphorus mobilization in early developing seedlings. Moreover, $\mathrm{Pi}$ contents in shoots and roots of BnPHT1;4 transgenic plants were remarkably higher than those in the controls under high and low Pi conditions, indicating that the high-affinity transporter BnPHT1;4 in roots may be responsible for Pi uptake from soil (Fig. 7c-d).

Additionally, rape seeds germinated on 1/2 MS medium with different $\mathrm{Pi}$ content. As shown in Additional file 1 Figure S5a, exogenous Pi did not affect the seed germination process of $B$. napus. There was no significant difference in germination rates of rape seeds among the different Pi conditions. On the other hand, post-germinated growth rate of seedlings in high Pi content was faster than that in Low Pi content. P deficiency significantly reduced the hypocotyl and root length of seedlings (Additional file 1 Figure S5b-d). The above results indicated that exogenous phosphorus don't affect seed germination but affect seedling growth of $B$. napus.

\section{Expression of BnPAP2 and BnPAP15 are altered in $B n P H T 1 ; 4$ transgenic plants}

Phosphorus stored in plant seeds is mainly in the form of phytic acid (myo-inositol hexakisphosphate). During 

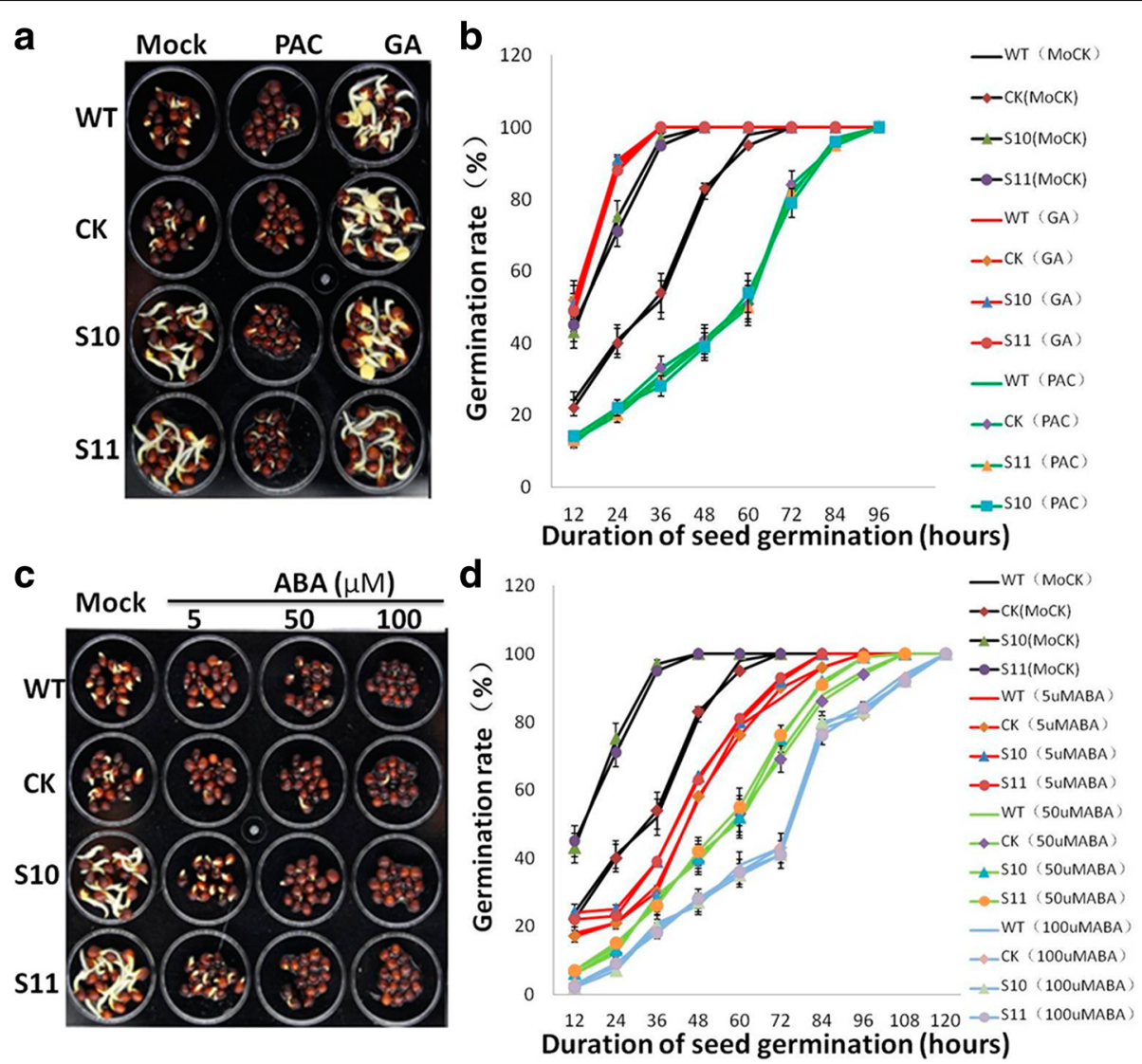

Fig. 6 Effects of GA and ABA on recovering the advanced-germination phenotype of the BnPHT1;4 overexpression transgenic Brassica napus seeds. (a and $\mathbf{b}$ ) effects of GA and PAC (paclobutrazol, a GA biosynthesis inhibitor) on seed germination. Seeds were treated with $0.1 \mathrm{mM}$ GA or $10 \mathrm{mM}$ PAC. One representative image per genotype (1.5 days after sowing) is shown in a. Quantitative analysis of germination rates are shown in b. The BnPHT1;4 transgenic seeds (S10 and S11) germinated more rapidly than those of the controls (WT and CK) under normal conditions (Mock). PAC delayed germination of the BnPHT1;4 transgenic seeds, while GA promoted seed germination of the controls. (c and $\mathbf{d}$ ) effects of ABA on seed germination. Seeds were treated with 5, 50 and $100 \mu \mathrm{M}$ ABA. One representative image per genotype (1.5 days after sowing) is shown in c. Quantitative analysis of germination rates are shown in d. ABA partially recovered the advanced-germination phenotype of the BnPHT1;4 transgenic seeds. The data are presented as means \pm SD $(n=5)$. WT, wild type; $C K$, transgenic null line; S10 and S11, independent BnPHT1;4 overexpression transgenic lines

seed germination, phytic acid is degraded by phytase to yield inositol and free phosphate provisions in early seedling development [29]. Therefore, there may be a link between Pi transporter and phytases. As shown in Fig. 8a-b, both BnPAP2 and BnPAP15 were up-regulated in the BnPHT1;4 overexpression seedlings of $B$. napus. The 5-bromo-4-chloro-3-indolyl phosphate (BCIP) staining also showed that the phosphatase activity were significantly increased in the BnPHT1;4 transgenic lines, compared with that in the controls (Fig. 8c). However, BnIPS1, BnACP5 and BnRNS1 were down-regulated in the BnPHT1;4 overexpression seedlings of B. napus (Fig. $8 \mathrm{~b})$. Moreover, pre-germinated seedlings grew on Pi-sufficient $(1 \mathrm{mM})$ medium for 7 days and then transferred to MS medium with different Pi contents for 2 days. Cotyledons of these seedlings were selected to investigate the expression of BnPAP2 and BnPAP15. As shown in Fig. 8d and Additional file 1 Figure S6, expression of BnPAP2 and BnPAP15 was induced in cotyledons of both transgenic lines and controls under Pi deficiency conditions. These results suggested that overexpression of BnPHT1;4 promotes P release from cotyledons of $B$. napus.

\section{Discussion}

It is well-known that the major phosphorus nutrient is restored in cotyledons of rape seeds [2]. Phosphorus nutrient provision in early developing seedlings depends on reallocated nutrient reserves before the root system have been established [30]. So, a P translocation system may exist in plants for transporting phosphorus from cotyledons to roots/hypocotyls during seed germination and early seedling development. So far, multiple P transporters involved in phosphorus uptake from soil and phosphorus translocation within the plant have been 

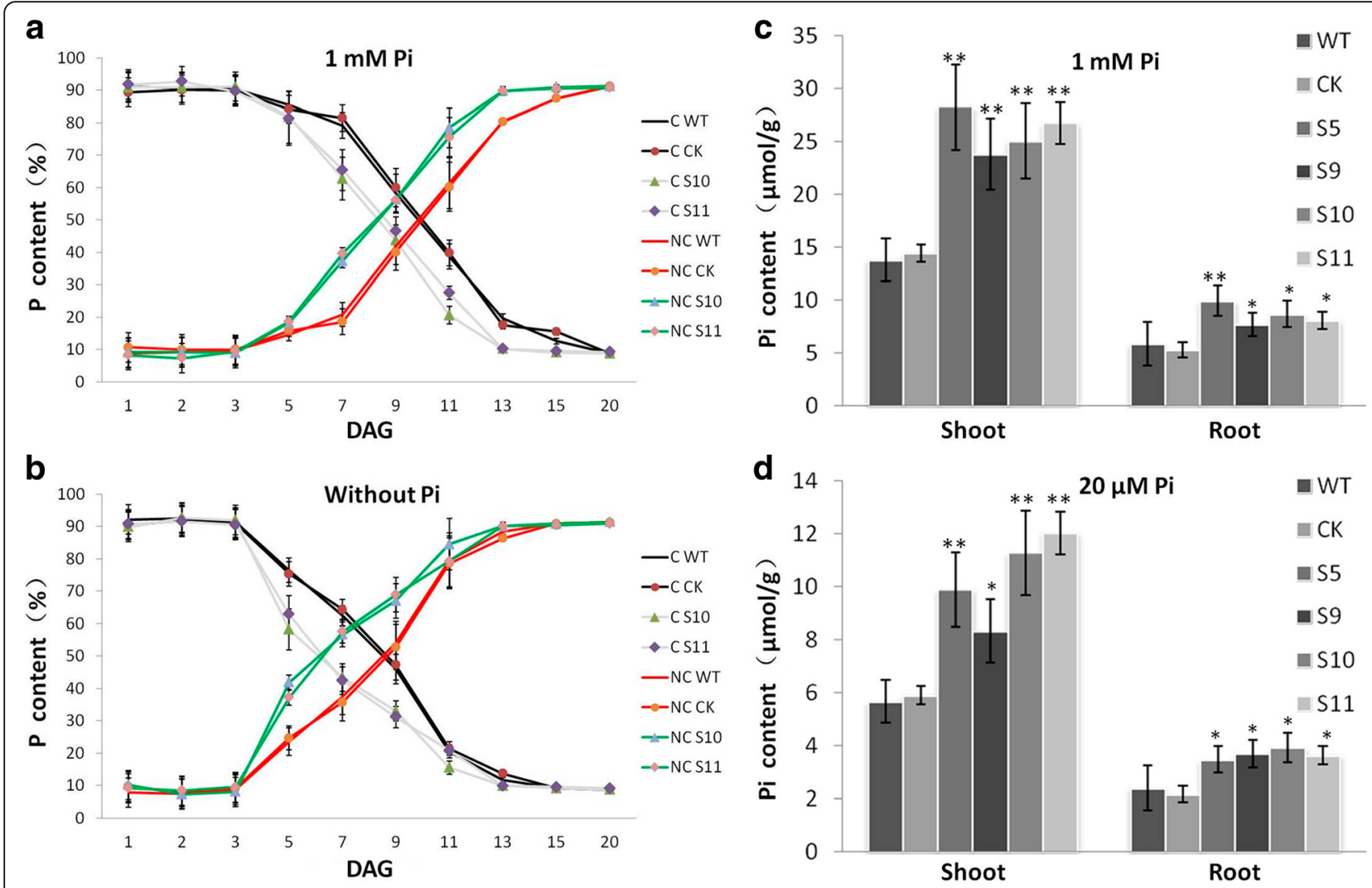

Fig. 7 BnPHT1;4 is involved in phosphorus mobilization in cotyledons and phosphorus uptake in roots of Brassica napus. (a and b) Dynamics of the relative pool sizes of the total phosphorus (P) in oilseed rape seedlings of the BnPHT1;4 overexpression transgenic lines and controls (WT and CK). Seedlings grew on 1/2 MS medium with $1 \mathrm{mM}$ Pi (a) or without Pi (b). P content was determined separately in cotyledons (c) and in roots plus leaves and hypocotyls (NC), and depicted as percentage of total P content in the whole seedling. The data are presented as means \pm SD $(n=3)$. (c and d) Phosphate (Pi) contents in shoots and roots of seven-day-old seedlings of the BnPHT1;4 overexpression transgenic lines and controls grown under $1 \mathrm{mM} \mathrm{Pi} \mathrm{(c)} \mathrm{and} 20 \mu \mathrm{M} \mathrm{Pi}$ (d) conditions. $\mathrm{KCl}$ was used to replace $\mathrm{KH}_{2} \mathrm{PO}_{4}$ in the medium for the equivalent amount of potassium. The error bars indicate the standard errors. Significance of difference was analyzed by Duncan's test $\left({ }^{*} p<0.05,{ }^{* *} p<0.01, n=9\right)$. WT, wild type; CK, transgenic null line; S5, S9, S10 and S11, independent BnPHT1;4 overexpression transgenic lines. DAG, day after germination

identified [31]. For example, AtPHT1 is involved in phosphorus uptake from soil, AtPHO1 transporters are responsible for loading $\mathrm{Pi}$ into xylem of roots and transferring phosphorus from the chalazal seed coat to the embryo in developing seeds, and AtPHT1;5 takes part in mobilizing stored phosphorus out of older leaves [3234]. However, the $\mathrm{P}$ transporter responsible for phosphorus translocation out of cotyledons during seed germination is not clear yet, especially in rape seeds. In this study, we found that BnPHT1;4 is strongly expressed in cotyledons during rape seed germination and early seedling growth in normal $\mathrm{Pi}$ conditions, suggesting that BnPHT1;4 functions in cotyledons for phosphorus transport. The transcripts of BnPHT1;4 are gradually increased in cotyledons after imbibition of the dry seeds and reach to its peak value before the cotyledons turn green, during which root system is not established for nutrient uptake.

In higher plants, the high-affinity P transport system is regulated by the status of phosphorus, different from the constitutively expressed low-affinity $\mathrm{P}$ transport system [35]. Previous studies revealed that Arabidopsis PHT1;1 and $P H T 1 ; 4$ are induced in roots under phosphorus deficiency [36], and BnPHT1;4 is highly expressed in P-starved roots [28]. Our data indicated that expression level of BnPHT1;4 is not changed in P deficient conditions when the cotyledons remain yellow, suggested that phosphorus nutrient provision in the early developing seedlings mainly depends on reallocated phosphorus reserves. On the other hand, BnPHT1;4 expression was significantly increased under P deficiency in both cotyledons and roots after the cotyledons turned green (Fig. 2). Induction of BnPHT1;4 expression by P deficiency in cotyledons and roots revealed that BnPHT1;4 may play an important role in both cotyledons and roots to cope with phosphorus limitations in the environment for seedling growth. Additionally, these results also suggested that the time of cotyledons turned green may be the critical point that phosphorus nutrient provision in germinated seeds and early developing seedlings mainly 


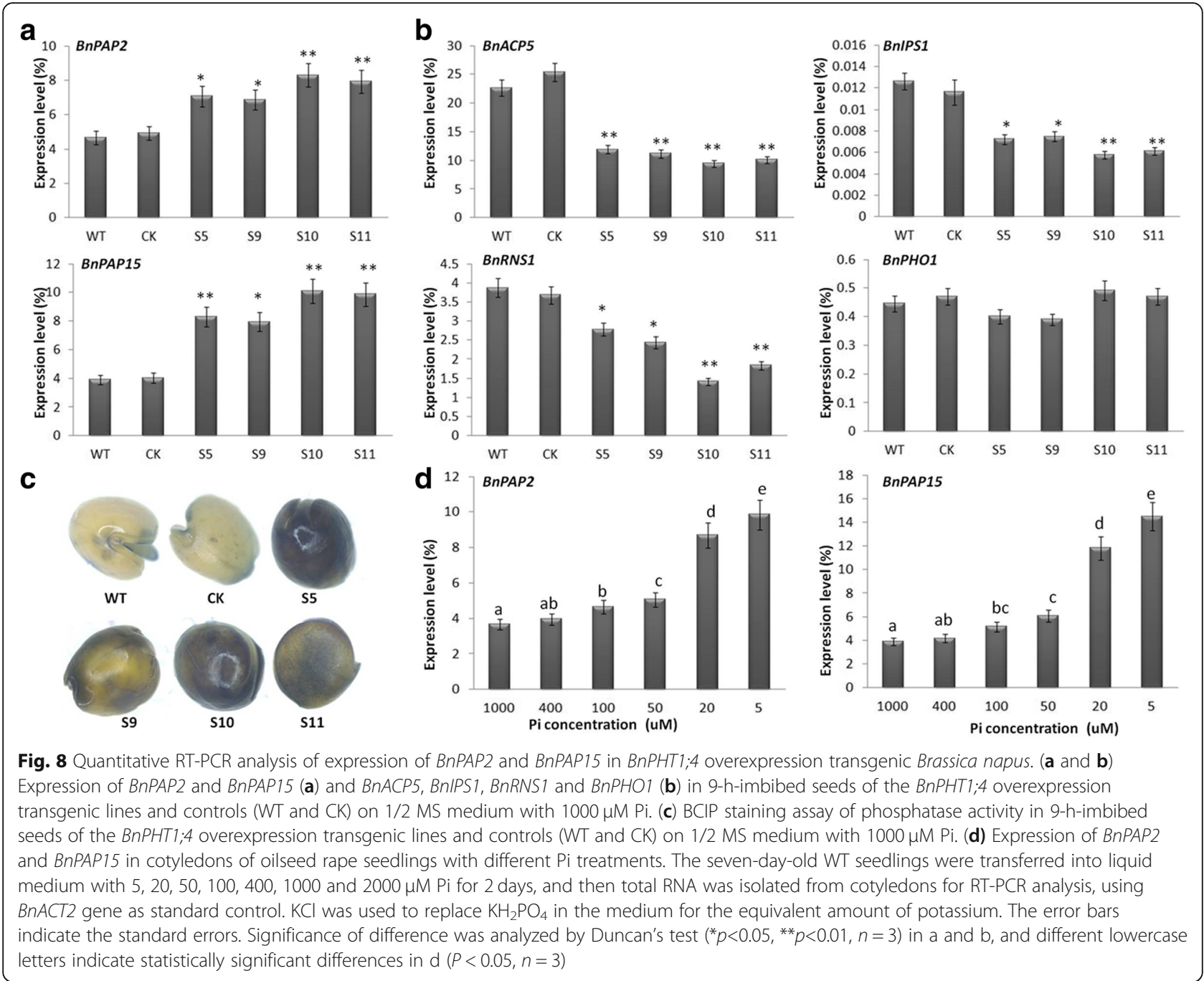

depends on reallocated phosphorus stored in cotyledons or acquire phosphorus from the rhizosphere.

The data of a time-course analysis showed that the total phosphorus content was constantly decreased in cotyledons of both BnPHT1;4 transgenic plants and wild type controls (Fig. 7). This is consistent with the results described by Eggert and colleagues [2]. The fact that phosphorus was consumed faster in cotyledons of oilseed rape seedlings without exogenous phosphorus supply indicated there is a $\mathrm{P}$ translocation system in cotyledons. Moreover, the total phosphorus content in cotyledons of BnPHT1;4 transgenic plants was decreased more rapidly than that in the controls when $P$ nutrition was supplied or deficient, indicating that BnPHT1;4 may be responsible for phosphorus translocation in cotyledons to provide phosphorus nutrient for early seedling development of $B$. napus. On the other hand, Pi contents in shoots and roots of the BnPHT1;4 transgenic seedlings were higher than that of wild type under high and low Pi conditions. And the hypocotyls and primary roots of the transgenic seedlings are significantly longer than those of the wild type controls. These results suggested that high-affinity transporter BnPHT1;4 may be responsible for phosphorus uptake from soil for seedling development. Also, our data suggested that BnPHT1;4 may perform dual functions in oilseed rape seedlings. It is responsible for phosphorus translocation in cotyledons before the cotyledons turn green and the root system is not established, and is responsible for phosphorus mobilization in cotyledons and phosphorus uptake in roots after the root system is established for nutrient uptake from soil.

It has been reported that seed germination is influenced by some endogenous factors (such as seed genotype, dormancy and hormone status) as well as some environmental conditions (such as moisture, oxygen and temperature) [37]. During seed germination, storage nutrients, such as lipids, proteins and others, are catabolized for maintaining early seedling development. Seed size and weight are correlated positively to seed germination and seedling establishment [38]. Larger canola 
seeds have higher levels of the measured mineral elements, in particular with those elements (such as K, P, S, $\mathrm{Mg}$ and $\mathrm{Mn}$ enriched in cotyledons, than those smaller seeds. A significant reduction in phosphorus content was found in those seeds that required more time for germination and seedling growth [2]. However, molecular mechanisms underlying the effect of phosphorus on seed germination and post-germinated growth of oilseed rape are still largely unclear in detail. In this study, our data revealed BnPHT1;4 may be responsible for phosphorus translocation in cotyledons during seed germination. Overexpression of BnPHT1;4 increased the phosphorus flow from cotyledons (source) to the most rapidly growing tissues and organs (sinks), thereby accelerating seed germination of $B$. napus.

Seed germination depends on the right phytohormonal response in the seed. Previous studies indicated that GA and $A B A$ are two primary endogenous factors regulating seed germination [17-20]. In this study, gene expression analysis and phytohormones measurement demonstrated that BnPHT1;4 positively regulates GA biosynthesis, but negatively modulates $\mathrm{ABA}$ biosynthesis in early developing seedlings, leading to an increase in the GA/ABA ratios (including GA1/ABA, GA3/ABA, and GA4/ABA). Moreover, exogenous GA could narrow the gap of germination velocity between the transgenic seeds and wild type controls, whereas the decreased endogenous GA level by PAC (a GA biosynthesis inhibitor) could partially recover the advanced-germination phenotype of the BnPHT1;4 transgenic seeds. Also, ABA could partially weaken this advanced-germination phenotype caused by overexpressing BnPHT1;4. Altogether, our data suggested that BnPHT1;4 may participate in regulating GA/ABA ratios to promote seed germination of $B$. napus.

\section{Conclusion}

In brief, BnPHT1;4 plays a critical role in phosphorus acquisition and mobilization to promote seed germination and early seedling growth of Brassica napus possibly by modulating ABA and GA biosynthesis. Our data presented in this study give a new insight into understanding the role of $\mathrm{P}$ transporter in B. napus and the effect of phosphorus on seed germination and post-germinated growth of oilseed rape. Also, this study provides a clue for further investigating the relationship of phosphorus and phytohormones ABA/GA balance during seed germination of B. napus.

\section{Methods}

\section{Plant materials}

Plants of oilseed rape (Brassica napus cv. Westar) and all the related transgenic lines grew in the experimental field in campus of Central China Normal University, Wuhan, China.
For seed germination phenotypic analysis, 100 seeds were cultured on a modified 1/2MS medium (containing $10.305 \mathrm{mM} \quad \mathrm{NH}_{4} \mathrm{NO}_{3}, \quad 0.75 \mathrm{mM} \quad \mathrm{MgSO}_{4}, \quad 9.395 \mathrm{mM}$ $\mathrm{KNO}_{3}, 1.495 \mathrm{mM} \mathrm{CaCl}, 3 \%(w / v)$ sucrose, and $0.8 \%(\mathrm{w} /$ v) agarose, $\mathrm{PH}$ 5.8) supplemented with different concentration of $\mathrm{Pi}$ in greenhouse $\left(24^{\circ} \mathrm{C}, 16 \mathrm{~h}\right.$ light $/ 8 \mathrm{~h}$ dark $)$. Additionally, $1.25 \mathrm{mM} \mathrm{KCl}$ was used to replace $\mathrm{KH}_{2} \mathrm{PO}_{4}$ in the medium for the equivalent amount of potassium. Then, 10, 50 and $100 \mu \mathrm{M}$ abscisic acid (ABA), $100 \mu \mathrm{M}$ gibberellins (GA) or $10 \mathrm{mM}$ paclobutrazol (PAC) was added in the medium according to the experiment requirements. Seed germination was considered when the radicle broke through the seed coat. For each germination test, five experimental replications were performed. Moreover, cotyledons, hypocotyls and roots at different developmental stages were harvested and used for length measurement, RNA isolation and P content assay. ABA, GA, and PAC (product number 33371) were ordered from Sigma-Aldrich Company Ltd., USA.

The oilseed rape seeds used in this study were provided by our lab.

\section{Vector construction and oilseed rape transformation}

The vector construction of pBI121-BnPHT1;4 and BnPHT1;4p:GUS is same with our previous report [28]. All constructs were transferred into Agrobacterium tumefaciens strain GV3101 that was then used for plant transformation. Transformation of oilseed rape ( $B$. napus) was carried out as described earlier [39]. The selective MS medium containing $50 \mathrm{mg} / \mathrm{L}$ kanamycin was used for screening the transformed seeds of B. napus, and the homozygous progeny plants (T3 and T4 generations) were used in the further experiments.

\section{Histochemical assay of GUS activity}

Histochemical assay of GUS activity was performed according to a modified protocol [40]. Imbibition seeds of BnPHT1;4p:GUS transgenic oilseed rape were collected at different time points after sowing under different Pi conditions. The samples were incubated in GUS reaction buffer containing 5-bromo-4-chloro-3-indolyl- $\beta$-dglucuronic aci d (X-Gluc) at $37^{\circ} \mathrm{C}$ for $12-48 \mathrm{~h}$. Then, $70 \%$ ethanol was used for clearing chlorophyll in the green tissues of seedlings. Images were documented using a Leica MZ16f stereomicroscope (Leica, Germany).

\section{Quantitative RT-PCR analysis}

Cotyledons and roots of imbibition seeds were collected at different time points after sowing, and total RNA was extracted using RNeasy Mini kit (Qiagen, German). Expression of genes was analyzed by real-time RT-PCR using the fluorescent intercalating dye SYBR Green in a detection system. A two-step RT-PCR procedure was performed according to a method described earlier [41]. 
All data were normalized against the expression level of the BnActin2 (BnACT2), and three biological replicates were performed for each experiment. The gene-specific primers used in the RT-PCR analysis are shown in Additional file 1 Table S1.

\section{Quantification of endogenous abscisic acid (ABA) and gibberellins (GA)}

The dry seeds and imbibed seeds were frozen in liquid nitrogen, ground to fine powder, and extracted with 10 $\mathrm{mL}$ extraction buffer (ethyl alcohol/hydrochloric acid). 8 ng D6-ABA and GA d2 isotope standards (OIChemIm Co. Ltd.) were added to plant samples before grinding. After oscillated at $4{ }^{\circ} \mathrm{C}$ for $30 \mathrm{~min}, 20 \mathrm{ml}$ dichloromethane were added to the crude extracts. Subsequently, the mixtures was centrifuged at $13,000 \mathrm{~g}$ for $5 \mathrm{~min}\left(4^{\circ} \mathrm{C}\right)$, and the lower organic phase was used in ABA and GA assay. After dried by nitrogen oxides and reconstituted, the lower organic phase was injected into a liquid chromatography-tandem mass spectrometry system consisting of an poroshell 120 SB-C18 reversed-phase column (Agilent, America) and a triple quadruple tandem mass spectrometer (Quattro Premier XE; Waters). Three biological replications were performed for each test.

\section{Measurement of phosphorus ( $\mathrm{P}$ ) and phosphate (pi) contents in plants}

Inorganic Pi measurements were performed as described previously [28]. The sample $(1 \mathrm{mg})$ was homogenized with $10 \mu \mathrm{L}$ extraction buffer $(100 \mathrm{mM} \mathrm{NaCl}, 10 \mathrm{mM}$ Tris, $1 \mathrm{mM}$ EDTA, $1 \mathrm{mM}$ phenylmethylsulfonyl fluoride and $1 \mathrm{mM} \beta$-mercaptoethanol). The homogenized sample was mixed with $1 \%$ glacial acetic with ratio of 1:9 ( V/ $\mathrm{V})$ and incubated at $42^{\circ} \mathrm{C}$ for $30 \mathrm{~min}$. After centrifuged at $13,000 \mathrm{~g}$ for $5 \mathrm{~min}, 300 \mu \mathrm{L}$ of the supernatant aliquot was added to $700 \mu \mathrm{L}$ of assay solution $(0.35 \%$ $\mathrm{NH} 4 \mathrm{MoO} 4,0.86 \mathrm{~N} \mathrm{H} 2 \mathrm{SO} 4$, and $1.4 \%$ ascorbic acid), and was incubated at $42^{\circ} \mathrm{C}$ for $30 \mathrm{~min}$. Pi content was measured on a TU-1901 UV-visible spectrophotometer with absorbance at $820 \mathrm{~nm}$ (Beijing Purkinje General Instrument limited liability company, Beijing, China). Plant total $\mathrm{P}(\mathrm{TP})$ content was analyzed by the molybdenum blue method using $\mathrm{H}_{2} \mathrm{SO}_{4}-\mathrm{H}_{2} \mathrm{O}_{2}$ to digest the samples at $300{ }^{\circ} \mathrm{C}$. Values were normalized to dry weight.

\section{BCIP staining assay}

Seeds were cultured on 1/2 MS medium containing $1 \mathrm{mM}$ $\mathrm{Pi}$. The samples were incubated in staining buffer containing 5-bromo-4-chloro-3-indolyl phosphate (BCIP) at room temperature for two hours. The commercial staining buffer (product number 0000325105) was purchased from Promega Company, USA.

\section{Additional files}

\begin{abstract}
Additional file 1: Figure S1. Expression profiling of BnPHT1;4 and BnPHT1;5 in 24-h-imbibed seeds of Brassica napus under Pi starvation. Figure S2. Expression profiling of BnPHT1;4 in shoots and roots of Brassica napus under Pi starvation. Figure S3. Assay of total phosphorus content in seeds of the BnPHT1;4 overexpression transgenic Brassica napus. Figure S4. Assay of thousand-grain weight of dry seeds of the BnPHT1;4 overexpression transgenic Brassica napus. Figure S5. The effect of exogenous phosphate ( $\mathrm{Pi}$ ) on seed germination and early seedling growth of Brassica napus. Figure S6. Quantitative RT-PCR analysis of expression of BnPAP2 and BnPAP15 in BnPHT1;4 overexpression transgenic Brassica napus. Table S1. Primers used in quantitative RT-PCR analysis. (PDF $464 \mathrm{~kb}$ )
\end{abstract}

Additional file 2: Dataset S1. Dynamics of the relative pool sizes (\%) of the total phosphorus (P) in the BnPHT1;4 overexpression transgenic seedlings of Brassica napus grown under $1 \mathrm{mM}$ Pi. Dataset S2. Dynamics of the relative pool sizes (\%) of the total phosphorus (P) in the BnPHT1;4 overexpression transgenic seedlings of Brassica napus grown without Pi. Dataset S3. Quantitative analysis of germination rates of the BnPHT1;4 overexpression transgenic seedlings of Brassica napus grown on 1/2 MS medium with 10, 50 and $100 \mu \mathrm{M}$ abscisic acid (ABA). Dataset S4.

Quantitative analysis of germination rates of the BnPHT1;4 overexpression transgenic seedlings of Brassica napus grown on 1/2 MS medium with $100 \mu \mathrm{M}$ gibberellins (GA) or $10 \mathrm{mM}$ paclobutrazol (PAC). (XLS $51 \mathrm{~kb}$ )

\section{Abbreviations}

ABA: Abscisic acid; BCIP: 5-bromo-4-chloro-3-indolyl phosphate; DAG: Days after seed germination; GA: Gibberellin; HP: P-sufficient; LP: P-deficient; P: Phosphorus; PAC: Paclobutrazol; PHT: Phosphate transporter; Pi: Phosphate; X-Gluc: 5-bromo-4-chloro-3-indolyl- $\beta$-dglucuronic acid

\section{Acknowledgements}

Not applicable.

\section{Funding}

This work was financially supported by the National Key R \& D Program of China (Grant No. 2016YFD0100202), and Project from the Ministry of Agriculture of China for Transgenic Research (Grant No. 2016ZX08009-003). Authors declare that none of the funding bodies have any role in the research design, the data collection and analysis, and the manuscript preparation.

\section{Availability of data and materials}

All data used during the current study are included in this published article or are available from the corresponding author on reasonable request.

\section{Authors' contributions}

$X \mathrm{BL}, \mathrm{FR}$ and $\mathrm{KLH}$ conceived and designed the research; KLH, HW, YLW, HXJ and $L Z$ performed the experiments; $K L H, Y Z$, FR and $X B L$ analyzed data, KLH and $X B L$ wrote the paper. All authors read and approved the final version of the manuscript.

Ethics approval and consent to participate

Brassica napus is a common crop extensively cultivated in the world. This study does not contain any research requiring ethical consent or approval.

Consent for publication

Not applicable.

Competing interests

The authors declare that none of the authors have any competing interests.

\section{Publisher's Note}

Springer Nature remains neutral with regard to jurisdictional claims in published maps and institutional affiliations. 
Received: 19 November 2018 Accepted: 8 April 2019 Published online: 25 April 2019

\section{References}

1. Finch-Savage WE, Bassel GW. Seed vigour and crop establishment: extending performance beyond adaptation. J Exp Bot. 2016;67:567-91.

2. Eggert K. Wire'n NV. Dynamics and partitioning of the ionome in seeds and germinating seedlings of winter oilseed rape. Metallomics. 2013;5:1316-25.

3. Osuna D, Prieto P, Aguilar M. Control of seed germination and plant development by carbon and nitrogen availability. Front Plant Sci. 2015;6:1023.

4. Cao MJ, Wang Z, Zhao QZ, Mao JL, Speiser A, Wirtz M, Hell R, Zhu JK, Xiang CB. Sulfate availability affects $A B A$ levels and germination response to $A B A$ and salt stress in Arabidopsis thaliana. Plant J. 2014;77:604-15.

5. Faried HN, Ayyub CM, Amjad M, Ahmed R, Wattoo FM, Butt M, Bashir M, Shaheen MR, Waqas MA. Salicylic acid confers salt tolerance in potato plants by improving water relations, gaseous exchange, antioxidant activities and osmoregulation. J Sci Food Agric. 2017;97:1868-75.

6. Hansch R, Mendel RR. Physiological functions of mineral micronutrients (cu, Zn, Mn, Fe, Ni, Mo, B, cl). Curr Opin Plant Biol. 2009;12:259-66.

7. Vance $C P$, Uhde-Stone C, Allan D. Phosphorus acquisition and use: critical adaptations by plants for securing a nonrenewable resource. New Phytol. 2003;157:423-47.

8. Misson J, Raghothama KG, Jain A, Jouhet J, Block MA, Bligny R, Ortet P, Creff A, Somerville S, Rolland N, Doumas P, Nacry P, Herrerra Estrella L, Nussaume $L$, Thibaud MC. A genome-wide transcriptional analysis using Arabidopsis thaliana affymetrix gene chips determined plant responses to phosphate deprivation. Proc Natl Acad Sci. 2005;102:11934-9.

9. Jouhet J, Marechal E, Block MA. Glycerolipid transfer for the building of membranes in plant cells. Prog Lipid Res. 2007;46:37-55.

10. Mimura T. Regulation of phosphate transport and homeostasis in plant cells. Int Rev Cytol. 1999;191:149-200.

11. Nussaume L, Maréchal E, Thibaud MC, Block MA. Plant plasma membrane and phosphate deprivation. Plant Cell Monogr. 2010;19:237-51.

12. Rausch C, Bucher M. Molecular mechanisms of phosphate transport in plants. Planta. 2002;21:23-37.

13. Gu M, Chen A, Sun S, Xu G. Complex regulation of plant phosphate transporters and the gap between molecular mechanisms and practical application: what is missing? Mol Plant. 2016;9:396-416.

14. Nagarajan VK, Jain A, Poling MD, Lewis AJ, Raghothama KG, Smith AP. Arabidopsis Pht1;5 mobilizes phosphate between source and sink organs and influences the interaction between phosphate homeostasis and ethylene signaling. Plant Physiol. 2011;156:1149-63.

15. Mudge SR, Rae AL, Diatloff E, Smith FW. Expression analysis suggests novel roles for members of the Pht1 family of phosphate transporters in Arabidopsis. Plant J. 2002;31:341-53.

16. Zhang F, Sun YF, Pei WX, Jain $A$, Sun $R$, Cao $Y$, Wu XN, Jiang $T T$, Zhang $L$, Fan XR, Chen AQ, Shen QR, Xu GH, Sun SB. Involvement of OsPht1;4 in phosphate acquisition and mobilization facilitates embryo development in rice. Plant J. 2015;82:556-69.

17. Finkelstein R, Reeves W, Ariizumi T, Steber C. Molecular aspects of seed dormancy. Annu Rev Plant Biol. 2008;59:387-415.

18. Graeber K, Nakabayashi K, Miatton E, Leubner-Metzger G, Soppe WJ. Molecular mechanisms of seed dormancy. Plant Cell Environ. 2012;35:1769-86.

19. Shu K, Liu XD, Xie Q, He ZH. Two faces of one seed: hormonal regulation of dormancy and germination. Mol Plant. 2016;9:34-45.

20. Nee G, Xiang Y, Soppe WJ. The release of dormancy, a wake-up call for seeds to germinate. Curr Opin Plant Biol. 2016;35:8-14

21. Shu K, Zhang H, Wang S, Chen M, Wu Y, Tang S, Liu C, Feng Y, Cao X, Xie Q. ABI4 regulates primary seed dormancy by regulating the biosynthesis of abscisic acid and gibberellins in Arabidopsis. PLoS Genet. 2013;9(6): e1003577.

22. Lee $S$, Cheng H, King KE, Wang W, He Y, Hussain A, Lo J, Harberd NP, Peng J. Gibberellin regulates Arabidopsis seed germination via RGL2, a GAI/RGAlike gene whose expression is up-regulated following imbibition. Genes Dev. 2002;16:646-58.

23. Koornneef M, Hanhart CJ, Hilhorst HW, Karssen CM. In vivo inhibition of seed development and reserve protein accumulation in recombinants of abscisic acid biosynthesis and responsiveness mutants in Arabidopsis thaliana. Plant Physiol. 1989;90:463-9.

24. Seo M, Hanada A, Kuwahara A, Endo A, Okamoto M, Yamauchi Y, North $H$, Marion-Poll A, Sun TP, Koshiba T, Kamiya Y, Yamaguchi S, Nambara E.
Regulation of hormone metabolism in Arabidopsis seeds: phytochrome regulation of abscisic acid metabolism and abscisic acid regulation of gibberellin metabolism. Plant J. 2006:48:354-66.

25. Frey A, Effroy D, Lefebvre V, Seo M, Perreau F, Berger A, Sechet J, To A, North HM, Marion-Poll A. Epoxycarotenoid cleavage by NCED5 fine-tunes $A B A$ accumulation and affects seed dormancy and drought tolerance with other NCED family members. Plant J. 2012;70:501-12.

26. Piskurewicz U, Jikumaru Y, Kinoshita N, Nambara E, Kamiya Y, Lopez-Molina L. The gibberellic acid signaling repressor RGL2 inhibits Arabidopsis seed germination by stimulating abscisic acid synthesis and ABI5 activity. Plant Cell. 2008;20:2729-45.

27. Frey A, Audran C, Marin E, Sotta B, Marion-Poll A. Engineering seed dormancy by the modification of zeaxanthin epoxidase gene expression. Plant Mol Biol. 1999;39:1267-74.

28. Ren F, Zhao CZ, Liu CS, Huang KL, Guo QQ, Chang LL, Xiong H, Li XB. A Brassica napus PHT1 phosphate transporter, BnPHT1;4, promotes phosphate uptake and affects roots architecture of transgenic Arabidopsis. Plant Mol Biol. 2014;86:595-607.

29. Hegeman CE, Grabau EA. A novel Phytase with sequence similarity to purple acid phosphatases is expressed in cotyledons of germinating soybean seedlings. Plant Physiol. 2001;126:1598-608.

30. Jahangir M, Abdel-Farid IB, Kim HK, Choi YH, Verpoorte R. Healthy and unhealthy plants: the effect of stress on the metabolism of Brassicaceae. Environ Exp Bot. 2009:67:23-33.

31. Młodzin'ska E, Zboin'ska M. Phosphate uptake and allocation - a closer look at Arabidopsis thaliana L. and Oryza sativa L. Front Plant Sci. 2016;7:1198.

32. Ullrich-Eberius $\mathrm{Cl}$, Novacky A, Fischer E, Lüttge U. Relationship betwee nenergy-dependent phosphate uptake and the electrical membrane potential in Lemna gibba G1. Plant Physiol. 1981;67:797-801.

33. López-Arredondo DL, Leyva-González MA, González-Morales SI, López-Bucio J, Herrera-Estrella L. Phosphate nutrition: improving low-phosphate tolerance in crops. Annu Rev Plant Physiol. 2014;65:95-123.

34. Vogiatzaki E, Baroux C, Jung JY, Poirier Y. PHO1 exports phosphate from the chalazal seed coat to the embryo in developing Arabidopsis seeds. Curr Biol. 2017;2(19):2893-900.

35. Furihata T, Suzuki M, Sakurai H. Kinetic characterization of two phosphate uptake systems with different affinities in suspension-cultured Catharanthus roseus protoplasts. Plant Cell Physiol. 1992;33:1151-7.

36. Shin H, Shin HS, Dewbre GR, Harrison MJ. Phosphate transport in Arabidopsis: Pht1;1 and Pht1;4 play a major role in phosphate acquisition from both low- and high-phosphate environments. Plant J. 2004;39:629-42.

37. Bassel GW. To grow or not to grow. Trends Plant Sci. 2016;21:498-505.

38. Weitbrecht K, Muller K, Leubner-Metzger G. First off the mark: early seed germination. J Exp Bot. 2011;62:3289-309.

39. Elahi H, Duncan RW, Stasolla C. Modification of oil and glucosinolate content in canola seeds with altered expression of Brassica napus LEAFY COTYLEDON1. Plant Physiol Bioch. 2016;100:52-63.

40. Xu WL, Zhang DJ, Wu YF, Qin LX, Huang GQ, Li J, Li L, Li XB. Cotton PRP5 gene encoding a proline-rich protein is involved in fiber development. Plant Mol Biol. 2013;82:353-65.

41. Li XB, Fan XP, Wang XL, Cai L, Yang WC. The cotton ACTIN1 gene is functionally expressed in fibers and participates in fiber elongation. Plant Cell. 2005;17:859-75.

\section{Ready to submit your research? Choose BMC and benefit from:}

- fast, convenient online submission

- thorough peer review by experienced researchers in your field

- rapid publication on acceptance

- support for research data, including large and complex data types

- gold Open Access which fosters wider collaboration and increased citations

- maximum visibility for your research: over $100 \mathrm{M}$ website views per year

At BMC, research is always in progress.

Learn more biomedcentral.com/submissions 